\title{
UNA NUEVA FILOSOFÍA DE LA ASTROLOGÍA EN LOS SIGLOS XII Y XIII: EL IMPACTO DE LAS TRADUCCIONES DEL ÁRABE Y LA POSTURA DE SANTO TOMÁS DE AQUINO
}

\author{
Luis Miguel Vicente García \\ Universidad Autónoma de Madrid
}

\section{RESUMEN:}

Se estudia en este trabajo la postura más abierta y conciliadora de la Iglesia, y de su pensador más universal en el siglo XIII, santo Tomás de Aquino, ante el problema de las estrellas. Tal apertura fue consecuencia de las traducciones de libros científicos durante los siglos XII y XIII y del legado árabe y judío.

Palabras claves: Santo Tomás de Aquino, astrología, traducciones de libros científicos, legado árabe y judío

\begin{abstract}
This article studies the Christian Church change of attitude during the XII and XIII centuries towards the problem of the stars and the effort of Saint Thomas Aquinas to build a new philosophy in order to assimilate the knowledge coming from the new translations of scientific works during that time. The Arabic and Jewish contributions were essential for such a change.
\end{abstract}

Key words: Saint Thomas Aquinas, Astrology, translations of scientific works, Arabic and Jewish contributions.

\section{EL LEGADO ÁRABE}

Como ha señalado Richard Lemay el papel desempeñado por la astrología en la ciencia y la mentalidad medievales es una laguna todavía en los estudios de historiografía, debido a una serie de prejuicios y circunstancias de todo tipo - sociales, históricas, conceptuales y culturales- que han obstaculizado la evaluación de lo que se entendía por astrología en la Edad Media. Para este crítico, la verdadera importancia de la astrología árabe en el pensamiento de la Europa medieval fue deformada a partir de la revolución científica de los siglos XVI y XVII que, con el descubrimiento de un nuevo orden del Cosmos y de unos métodos de observación más precisos, hizo caer a la astrología medieval en olvido. Por otra parte, el interés de los historiadores, influidos por un favoritismo renacentista hacia los clásicos griegos, ha motivado que el énfasis, al estudiar la astrología medieval, recayera fundamentalmente en el Tetrabiblos de Ptolomeo, mientras que se han descuidado las obras de Aristóteles, por el lado de la tradición griega, y de Albumasar, por parte de la tradición árabe, mucho más influyentes, a juicio también de Jean-Claude Vadet (52), para formar el bagaje de conocimientos astrológicos durante la Edad Media y el Renacimiento. ${ }^{1}$ Fue relativamente reciente la atención de la crítica hacia 
las traducciones arabo-latinas como punto de referencia imprescindible para un conocimiento de la cultura medieval: Millás Vallicrosa (»El literalismo» 155) cita como pionero a Jourdain con su estudio de 1843. No se trata de que las obras de Aristóteles no se conocieran, sino de que no se ha estudiado lo suficiente la relación de De caelo, De generatione et corruptione, o de la Metereologica con la astrología científica, a pesar de que la cosmología y la física de Aristóteles fueran la base de la creación y difusión de las doctrinas astrológicas y el salvoconducto que permitió - por el prestigio del filósofo- que se aceptaran los tratados árabes en Occidente:

To a careful observer of the evolution of science after Aristotle, there can be little doubt that these works supplied the scientific background of astrology during 2000 years after $\mathrm{him}$. And therefore astrology, during all that time, formed an indespensable and intimate part of physical science and cosmology. Until the sixteenth century, no scientist could ever afford, or even be tempted, to reject Aristotle's ground principles of physics: Aristotle, for all of them, was science incarnate [...] Hence the effort to understand medieval attitudes toward astrology by applying to this science our contemporary paradigm of science [...] seems to foreclose in advance all avenues leading to the medieval mind, to its structural framework, and to the content of its own different paradigm (Lemay 58).

Pero además de los prejuicios que desató la revolución científica de los siglos XVI y XVII, existió también, desde la antigüedad, la oposición de un sector importante de la Iglesia que veía en la astrología, enseñada bajo nombres musulmanes, una constante amenaza; la astrología, en el mundo cristiano se sentía como algo adquirido de las gentes orientales o de la filosofía pagana. En el mundo árabe, en cambio, la vemos ligada desde el principio - como en el caso del pionero Albumasar - a los palacios califales. La astrología fue en el mundo árabe un intento de conciliar fe y razón, hasta el punto de que se pudieran saber los designios de Dios, utilizando la razón, si se era lo suficientemente sabio y se aprendía a leer en el mapa de los cielos. La formación del astrólogo es enciclopédica, y su objetivo es integrar los varios saberes en una ciencia que pueda dar cuenta de la totalidad de los aspectos humanos y naturales. Eso es posible porque se concibe el universo como una sola maquinaria regida por sus leyes de cambio, las cuales no se pueden desligar de lo humano ni de las manifestaciones del espíritu:

Ce qui fait la force de l'Astrologie [...] c'est qu'elle restitue l'Unus Mundus à la conscience déchirée des grandes crisis de civilisation. Elle le restitue doublement: en présentant un système d'integration [...] mais aussi, [...] en courrant comme le fil de colier qui relie les perles des civilisations au cours de multiples exaltations et de multiples déclins cultureles (Duran 201).

Por otra parte, la revolución en las ideas astrológicas no nació sólo de la especulación, sino que la superioridad árabe en materias técnicas y artesanales hizo de los musulmanes los verdaderos maestros del astrolabio. ${ }^{2}$ Albumasar florece en la espléndida Bagdad califal del siglo IX, y aparece allí envuelto en la construcción de un importante observatorio, cerca de una ciudad babilónica antigua. ${ }^{3}$ Ese desarrollo técnico les permitía, además de sus preocupaciones más coloridas y profundas, un conocimiento del cielo mucho más preciso que el que tenían los griegos. El astrolabio árabe permitió popularizar la astrología porque su utilización no requería conocimientos astronómicos, ${ }^{4}$ de la misma manera que hoy usar un computador no implica tener conocimientos de cibernética.

\footnotetext{
Cf. Lemay 57.

Cf. Vadet 52.

Cf. Halbronn 210.

Cf. Vedet 53.
} 
El papel del astrólogo árabe Albumasar fue de más trascendencia que el de Ptolomeo porque fundía en sus teorías astrológicas la herencia de la física aristotélica con los conocimientos de astrología heredados de los alejandrinos, de los persas, de los babilonios y de los indios. Esta ciencia, expandida por los numerosos astrólogos árabes influidos por Albumasar, desempeñará un papel fundamental en la sociedad medieval ya que todo aspecto de la vida tiene cabida en su competencia, desde la política a las relaciones familiares, pasando por la medicina; la astrología, como bien ha señalado Otto Wedel, «offering, as it did, a reasoned explanation of an infinite diversity of physical phenomena, and including in its scope psycology and ethics, made possible even in the Middle Ages dreams of a universal science» (iii). La carta astral ilustraba la concepción del todo, simbolizado en el círculo, entre cuyas partes, por el principio de la similitud o la simpatía, se daba una estrecha correspondencia:

C'est sur cette propiété fondamentale de <langage intégral > que repose le fait qu'à toute figure (proportions ou situations géométriques) donnée par l'astronomie, correspond un sens donné à l'événement humain [...] Le langue de l'astrologue n'est qu'une cas particulier, par figures astronomiques interposées, de la numérologie pythagoricienne et hermétise. Aux nombres et leurs combinations, aux configurations nombrables que donne la «carte» astronomique, correspondent les qualités de la destinée humaine (Durand 204206).

El principal libro de Albumasar, el Introductorium maius in astronomiam, fue traducido al latín por Iohanis Hispanus en1133 y por Herman de Carinthia - Herman de Dalmacia para Otto Wedel (55) - en 1140. Es de carácter enciclopédico y amalgama, como se ha dicho, la astrología de varias tradiciones con las teorías peripatéticas. ${ }^{5} \mathrm{~J}$. D. North se ha ocupado de estudiar las nociones aristotélicas que dieron pie a las expansiones astrológicas sobre la teoría del movimiento. Aristóteles da prioridad al Sol en sus explicaciones, mucho menos a la Luna y menciona raramente a los planetas:

In giving an account of the change in the world, Aristotle has said virtually nothing about the planets (as opposed to the luminaries, the Sun and the Moon). Albumasar thought that they, the planets, offered the key to the enormous diversity change in our world (7).

La traducción de Herman de Dalmacia contiene invenciones propias como la predicción del nacimiento virginal de Cristo; ${ }^{6}$ la de Iohanis Hispanus suministró nueva terminología en latín y diferencia claramente entre los conceptos de astronomía, como la ciencia de los movimientos celestes, y astrología o la ciencia de los juicios de las estrellas. Lemay explica el origen del término «juizios» debido a la traducción de Iohanis Hispanus; su equivalente más aproximado a la palabra original árabe debería ser «auctoritates;» la traducción «juizios» sería la responsable de la expresión «astrología judiciaria.» ${ }^{7}$ En cuanto a la trascendencia de Albumasar en Occidente baste citar a modo de ilustración que Alanus de Insulis hacia 1190 considera a Albumasar en su Anticlaudianus como la autoridad en astrología y a Ptolomeo la autoridad en astronomía. ${ }^{8}$ Sobre las bases que estableciera Albumasar se desarrolla entre los árabes una astrología que tuvo grandes continuadores, entre los más destacados por sus repercusiones en la Europa medieval y renacentista, se encuentran Abenragel y Alcabitius. Para Wedel los tratados científicos de Albumasar, Abenragel y Alcabitius presentan un marcado contraste con la astrología clásica que, como la de Ptolomeo, se dedica exclusivamente a la predicción del futuro de acuerdo con la posición de las estrellas en la hora del nacimiento (53). El siste- 
ma de interrogaciones y elecciones predomina, en cambio, en las obras de estos astrólogos árabes. Este tipo de astrología implica una serie de reglas mediante las cuales el astrólogo contesta las posibles preguntas de un cliente - interrogaciones - sobre aspectos diversos -identidad de un ladrón, el paradero de un objeto perdido, etc-; o establece el momento favorable —elecciones - para el desarrollo de una actividad — boda, decisión política, batallas, etc. Respecto a la astrología judiciaria, el sistema de elecciones e interrogaciones, no predice en sí el futuro sino que establece una serie de condiciones favorables o desfavorables y por tanto evita el fatalismo que acompaña al sistema ptolemaico; ${ }^{9}$ la medicina astrológica lo adaptó enseguida. Sin embargo, en la astrología judiciaria árabe también se incluía el levantamiento de horóscopos - las natividades. La división de la astrología judicial comprende conjunciones y revoluciones, natividades, interrogaciones y elecciones. ${ }^{10}$ La más importante distinción, en todo caso, entre la astrología ptolemaica y la árabe, la expresan las siguientes palabras de Vadet:

Mieux que les Grecs dont leur système est issu, les premieres astrologues de l'Islam auront tendu a présenter l'astrologie sous la forme d'un beau livre d'images écrit de la main de Dieu et où sont consignées les grandes lignes de l'espace et du temps que la Providence prend soin de rendre intelligibles à ceux dont elle assure harmonieusement le gouvernament. La voûte céleste est bien alors le vestibule de la Cité divine» (59).

El modo de considerar la astrología entre los miembros de una religión monoteista como el Islam era mucho más apto para ser asimilado por el cristianismo que la astrología griega. Averrores, Maimónides o Santo Tomás, se enfrentan de un modo parecido al tema de los astros. Esta ciencia constituía un sustrato común y una materia de colaboración en la época de Alfonso X, sólo eclipsada por la intransigencia mutua en materia de cuál era la auténtica revelación.

Albumasar en su Introductorium, después de exponer los principios de astrología, hace una defensa de ésta apoyándose en la teoría aristotélica de la quinta esencia, o sea, que la sustancia de los cuerpos celestes no está compuesta de los cuatro elementos que forman el mundo sublunar porque, si así fuera, estarían sometidos a los mismos cambios que los cuerpos terrestres. Las estrellas, pues, compuestas de una sustancia diferente, son esféricas, trasparentes y tienen un movimiento circular perpetuo; el movimiento en la esfera sublunar, en cambio, es de generación y corrupción constantes. El Sol y la Luna sobre todo causan la moción sublunar en tanto que el principio de permanencia se origina en las estrellas fijas. Albumasar sigue estas doctrinas, aunque las adapta otorgando a los planetas el dominio sobre los detalles de la vida terrestre mientras que a las estrellas fijas les atribuye un dominio más general en proporción a su mayor lentitud: su idea es que cuanto más veloz se mueve un planeta más influye en los cambios terrestres, mientras que la esfera de las estrellas fijas circunda la tierra con un movimiento perpetuo, sin alterar su posición ni su distancia respecto a la Tierra. Los siete planetas, en cambio, se mueven rápidamente y sigue cada uno su propio curso, razón por la que los cambios de la región sublunar no pueden cesar. Observando las mudanzas en los cursos de esos planetas, puede el hombre comprender la variedad del cambio en el mundo. Respecto al problema del fatalismo que se asociaba con la astrología, Albumasar busca una solución de compromiso parecida a la que adoptará Tomás de Aquino. El astrólogo árabe distingue entre los hechos contingentes y los hechos necesarios, que son los que pueden predecirse. Todas las cosas necesarias están regidas por las esferas celestes; pero el hombre, compuesto de un alma racional, puede elegir y gobernar el cuerpo. ${ }^{11}$ Sin embargo, como señala Wedel, la respuesta de 
Albumasar ofrece cierta dificultad para resolver el determinismo, ya que también considera como Aristóteles que las estrellas están dotadas de un alma racional - cosa que no habían admitido ni Plotino ${ }^{12}$ ni los Santos Padres ${ }^{13}$ - y poseen en consecuencia el poder de afectar la armonía entre el alma y el cuerpo. La Iglesia dio expresión oficial a su condena del animismo del mundo en el concilio de Sens de $1141 .{ }^{14}$ Pero la fusión de la cosmología aristotélica con la nueva ciencia árabe, y las distinciones de Albumasar sobre las cosas contingentes y las necesarias abrían el camino para encontrar una respuesta de compromiso entre la astrología y la Iglesia, como veremos en Santo Tomás.

\section{LA ASTROLOGÍA EN LA ESPAÑ̃ MUSULMANA}

La España arabigoandaluza fue, en todo caso, la responsable de las traducciones de obras científicas árabes al latín y al castellano -también al hebreo-durante los siglos XII y XIII. El ilustre historiador de la ciencia Juan Vernet señala cómo desde el momento de la conquista, la lengua árabe fue introduciéndose entre los cristianos y cómo figuran muy tempranamente glosas árabes al margen de los códices visigodos. ${ }^{15} \mathrm{~A}$ principios del siglo $\mathrm{X}$ sitúa este crítico la llegada a Al-Andalus de una serie de ideas esotéricas, con carácter científico y filosófico, que fueron reunidas en una obra titulada Epistolas de los Hermanos de la Pureza, introdudidas en Córdoba por Maslama de Madrid. ${ }^{16}$ En estas cartas - traducidas al alemán en el siglo pasadose establece la división de las ciencias matemáticas en cuatro disciplinas: aritmética, geometría, astronomía y música: «se nos da la enumeración correcta del quadrivium cuyo origen remoto hay que buscar en Arquitas de Tarento y el inmediato, en San Agustín, Boecio y Ammonio» (Vernet La ciencia 69). Esta obra, de carácter enciclopédico, tuvo una inmensa divulgación y a mediados del siglo XI la citan los poetas como Mosé Ibn Ezra. La obra, en cincuenta epístolas, difunde la ciencia vista a través del neoplatonismo y del pitagorismo. ${ }^{17} \mathrm{El} \mathrm{im-}$ pacto de estas cartas en el pensamiento y la literatura medievales en España ha sido ilustrado sobre todo por Francisco Rico, para quien:

Nunca antes y quizá nunca después la doctrina del microcosmos se planteó tan radical, exhaustiva y sistemáticamente como en las Rasa' il Ijwan al-Safa' [Cartas de los Hermanos de la Pureza] [...] apenas hay sección de la enciclopedia en que cada aspecto de la naturaleza, la vida social, la cultura o la religión no se explique por referencia al paralelismo de macrocosmos y microcosmos, clave de todo conocimiento (62). ${ }^{18}$

Rico rastrea las huellas de esas cartas en los Libros del saber de astronomía, en la Poridat, en La doncella Teodor y en las Partidas, y advierte al lector que esos ejemplos solos «no dan aún idea adecuada del influjo de las Rasa'il en las letras peninsulares» (76). Tal enciclopedia es, en todo caso, una derivación de las teorías astrológicas que muestran fascinación por el mapa de correspondencias entre el mundo sublunar y supralunar tal y como lo había esta-

12 Cf. Luis Miguel Vicente. «Plotino y el problema de las estrellas: una solución para los neoplatónicos» Revista Española de Filosofia Medieval. Universidad de Zaragoza, 2000 vol. 7, pp. 191-198.

13 Cf. Luis Miguel Vicente. «San Agustín, San Gregorio y San Isidoro ante el problema de las estrellas: fundamentos para el rechazo frontal de la Astrología» Revista Española de Filosofía Medieval. Universidad de Zaragoza, 2001: vol. 8, pp. 187-205.

14 Cf. Rico 69-70.

15 Cf. Vernet Historia 56.

16 Cf. Rico 61, Cruz Hernández 1 148-152, Vernet La ciencia 69.

17 Cf. Vernet Historia 59

18 No transcribo los rasgos diacríticos de la lengua árabe o hebrea. 
blecido Ptolomeo a quien se cita con bastante frecuencia. ${ }^{19}$ Las Cartas, apoyadas en parte en el conocimiento establecido por los astrólogos, no ponen el énfasis en la parte operativa de la astrología, sino en las implicaciones filosóficas que podían extraerse de una concepción del mundo tan regular y geométrica como la que sostenía la ciencia de los astrónomos y astrólogos. Las teorías emanantistas que transmiten se entroncan, por otro lado, con la tradición neoplatónica y su teoría de la cadena de la evolución.

La vinculación de esas Cartas al desarrollo de la astronomía-astrología en Al-Andalus es necesaria para entender su filosofía que no puede ser explicada solamente como una evolución de las corrientes filosóficas de la antigüedad. El esplendor del califato de Córdoba se manifestó por su lado cultural en la creación de una Biblioteca de Palacio que constaba de cuatrocientos mil volúmenes y comprendía obras traducidas del griego, otras procedentes de Oriente y las traducciones latino-árabes que encargara el príncipe heredero al-Hakam $\Pi{ }^{20}$ En cuanto al desarrollo de la astronomía en la España arabigoandaluza, en el siglo IX se introducen las teorías astronómicas indias de Sindhind, se construye un planetario y un reloj; ${ }^{21}$ la astrología fue usada profusamente durante el califato de Córdoba, en general ligada, como en el caso de los judíos, a la sensación de que los astros anunciaban el fin próximo del califato:

Los terrores milenarios de la cristiandad tuvieron su equivalente en el mundo arábigo-español en los signos celestes, que según los astrólogos cordobeses anunciaban el inminente fin del califato. Córdoba fue testigo del eclipse de sol (1004); luego apareció un cometa (1006), y para terminar tuvo lugar la conjunción Júpiter con Saturno en el signo de Virgo. De todos estos acontecimientos, los astrólogos dedujeron que estallaría la guerra civil, y del último, en concreto, que por ocurrir en un signo bifaz, los soberanos que gobernaran durante la misma tendrían dos reinados distintos: y así fue» (Vernet Historia 60).

Vernet comprueba con los métodos de la astronomía histórica que esos fenómenos ocurrirían por las fechas que dicen las crónicas (Historia 61-62). Hacia la segunda mitad del siglo $\mathrm{XI}$ destaca Azarquiel, en Toledo. Este se inicia en la construcción de instrumentos para los astrónomos y realiza varios descubrimientos importantes para la revolución astronómica renacentista. Su mérito fue haber sabido unir las prácticas de la astrología a las consideraciones de la matemática pura. ${ }^{22}$

En la época de la invasión de los almorávides y almohades tres figuras destacan: Avenpace -quien propone una renovación del sistema planetario, con objeciones al sistema de Ptolomeo, que violaba, según el astrónomo árabe, varios principios peripatéticos. También Averroes y Maimónides critican el sistema ptolemaico basándose en Aristóteles. ${ }^{23}$ Junto a él destacan Avenzoar, que sobresalió más en el campo de la medicina, y cerrando el siglo XII, Averroes (1126-1198), cuya fama fue inmensa tanto entre los cristianos como entre los árabes y del que Vernet ha escrito: «es posiblemente el español que mayor influjo ha ejercido en el pensamiento humano» (Historia 67). Su aportación principal se debe a los comentarios a las obras de Aristóteles (sobre todo a la Fisica y al De caelo) que, como hemos visto al hablar de Albumasar, constituyen un sustrato indispensable para la aceptación de la astrología en el Occidente cristiano. A la labor de Averrores hay que añadir simultáneamente, en una línea de pensamiento parecida, la de Maimónides (1135-1204) en el mundo judío, de quien nos ocupare-

19 Miguel Cruz Herñández dice que se cita a Ptolomeo veintiséis veces directamente y otras dicecisiete al mencionar el Almagesto; tambíen se cita a Plotino, a Arsitóteles — sobre todo en la terminología-, Euclides, Hipócrates y por supuesto Platón, entre los más destacados (1 151).

20 Cf. Vernet Historia 59.

21 Cf. Vernet Historia 57

22 Cf. Vadet 51.

23 Cf. Vernet La ciencia 98 y ss. 
mos más adelante. El florecimiento de los saberes científicos en la España musulmana no decae hasta las Navas de Tolosa (1212), momento que marca, de otra parte, la adquisición para los cristinanos, como fruto de la Reconquista, de un ingente corpus de obras árabes que registraban sus progresos científicos. Alfonso $X$ los hizo traducir, sirviéndose sobre todo de sus colaboradores judíos. Para la historia de la cultura medieval española - y para la de la Europa cristiana- ese hecho fue capital aunque, en términos de ciencia, como bien señala Vernet, «los cristianos peninsulares [...] no supieron continuar el desarrollo lógico que llevaba implícito en sí la brillante civilización arabigo-andaluza» (Historia 70).

\section{EL ELEMENTO JUDÍO}

La eclosión de obras astrológicas traducidas primero del árabe al latín y más tarde, bajo el reinado de Alfonso X, al castellano directamente, no se explica si no es por la presencia del elemento judío como mediador entre la cultura árabe y la latino-castellana. Sin embargo, la tarea de los judíos tuvo mucho más relevancia que la de meros mediadores entre ambas culturas. En cuanto a su papel en el desarrollo de la astrología y la filosofía en la Europa occidental, los datos históricos hablan por sí mismos: los judíos contribuyeron de un modo muy destacado al desarrollo de las escuelas astrológicas árabes, así como a la resurrección del neoplatonismo, sobre todo de Plotino, en el que se inspira la obra de Avicebrón - Salomon ben Gabirol- La fuente de la vida, que traducida al latín en el siglo XII por Johannus Hispanus, fue la base del neoplatonismo renacentista. Avicebrón influye también incluso a los pensadores escolásticos cristianos, como Santo Tomás, que lo cita en sus obras a menudo. Ibn Gabirol adopta el sistema de la emanación de Plotino: se trata de concebir el universo compuesto por sustancias inferiores que emanan de las superiores de igual modo que la luz se propaga debilitándose a través del espacio. Lo que tienen en común las distintas sustancias es la materia, y se diferencian por la forma; el mundo de la materia se divide en mundo sublunar y translunar. ${ }^{24}$ Avicebrón influido por el tamiz de su contexto judío, adapta las teorías de Plotino y las hace más asimilables para el pensamiento cristiano, ya que en la concepción emanantista que incorpora de Plotino, la acción creadora de un Dios personal, que caracteriza a las religiones de las tres sectas, es la responsable de dar unidad y causa primordial a la organización del universo; teoría que se asemeja a la de los Santos Padres, ya que aquél como éstos tratan de integrar fe - una fe confesional, no intelectual como en Plotino- y ciencia. La astrología, en este sentido, sirvió de punto de contacto supra-confesional, entre los sabios de las tres religiones, cuya comunicación, cuando se trataba de religión, estaba limitada por las exigencias literalistas de su respectivo credo, que no les permitía sino diatribas y ataques mutuos. La astrología, en cambio, ofrecía un punto de contacto posible para intercambiar conocimientos. Los principios astrológicos, según se consolidaron entre sus cultivadores de las tres castas, fueron supeditados a la acción del concepto de Dios de sus respectivas religiones, y eso se manifiesta en el uso, en obras astrológicas, de clichés abundantísimos, sobre todo cuando se pronostica, expresando que el vaticinio ocurrirá sólo si Dios lo permite. Los clichés o fórmulas en las que se invoca la ayuda y el asentimiento de Dios, servían en cualquiera de las tres religiones, y en las traducciones, lo único que da la clave de qué Dios se trata —el musulmán, el judío o el cristiano- es el contexto; de modo que en la traducción del Libro conplido, por ejemplo, Abenrragel se refiere a Alá, pero en la versión cristiana, esa fórmula de referencia se entiende como el Dios cristiano. De ese modo, la astrología ofrecía una especie de lengua franca para el conocimiento y la especulación en la sociedad intercastal de la España Medieval. 
Con la invasión e intrasigencia religiosa de los almohades en la primera mitad del siglo $\mathrm{XII}$, la cultura hispanohebrea se desplaza desde Andalucía y el resto de la España árabe, hacia los estados cristianos de la Península, sobre todo a las capitales Toledo y Barcelona. ${ }^{25}$ Tras este éxodo, los judíos españoles, dada su avanzada cultura y sus dotes lingüísticas privilegiadas, empezaron a ejercer funciones de administración pública en las cortes cristianas:

En ellos encontraron los soberanos españoles de la Reconquista técnicos colaboradores que, perfectamente impuestos de los métodos y posibilidades de la zona de población recién conquistada, iban a ser órganos muy adecuados de la administración pública. Así es que, con raras excepciones, los encontramos en las cortes de los sucesivos monarcas de Castilla y Aragón, en calidad de almojarifes, bailes, administradores de rentas o tesoreros, alfaquíes, secretarios de correspondencia árabe, astrólogos, truchimanes; ellos eran además, los grandes arrendatarios de las rentas, impuestos y alcabalas del fisco (Millás Vallicrosa Literatura 112). .

La presencia de los judíos fue especialmente importante en los reinados de Fernado III el Santo y Jaime I el Conquistador, que coinciden con el apogeo de la labor de Reconquista. Con Alfonso el Sabio los judíos intervienen profusamente en las actividades culturales e influyen de una manera decisiva en la gran enciclopedia Libro del saber de astrología. ${ }^{26}$ Como parte de esa empresa se tradujo el Libro conplido en los iudizios de las estrellas, que fue, de los libros alfonsíes el de más trascendencia para la cultura astrológica medieval europea ${ }^{27}$ Los judíos solían colaborar en la traducción de obras del árabe al romance; traducciones, como Millás Villacrosa ha señalado, muy literales y llenas de arabismos (»El literalismo...»). Los judíos, antes que los árabes, son los responsables de la introducción de la nueva ciencia astrológica en la Europa cristiana y no la labor aislada de Abelardo de Bath, a quien Otto Wedel propone como el introductor de la ciencia árabe en Occidente. ${ }^{28}$ Es verdad que Abelardo de Bath, nacido hacia 1100 en Inglaterra e incansable viajero por el Oriente, ofrece testimonios muy tempranos de asimilación de la nueva ciencia árabe y de la importancia que, a raíz de ese hecho, toma la astronomía en Europa. En una de las obras independientes de este autor, De eodem et diverso se hace una descripción de las siete artes liberales representada por una procesión de siete vírgenes en la que se sitúa a la Astronomía a la cabeza de las demás, rodeada de esplendor y portando en su mano derecha un cuadrante y en su izquierda un astrolabio. La astronomía describe la forma del mundo, el curso y las órbitas de los planetas y la posición de los signos junto con todos los detalles y medidas de los cuerpos; o sea, se identifica con el conocimiento del mundo tanto en presente como en pasado y futuro ya que los seres del mundo superior son los causantes de lo que ocurre en el inferior. ${ }^{29}$ No podemos tomar, sin embargo, la labor de Abelardo de Bath como pionera en la introducción de la nueva ciencia árabe en Occidente. Millás Vallicrosa, en su famoso trabajo Assaig d'història de les idees físiques i matemàtiques a la Catalunya medieval, estudió la influencia de la ciencia árabe matemático-astronómica en la cultura cristiana de los siglos X al XIII y demostró, entre otras cosas, que en un manuscrito latino sobre Aritmética de Boecio, ya aparecen notas marginales en árabe. Pero su contribución más destacada en esa obra son los estudios sobre los textos científicos que se conservan en el Mo-

25 Cf. Millás Vallicrosa Literatura 112 y ss.

26 El título Libros del saber de astronomia fue elegido por Rico y Sinobas en su editio princeps. Preferimos emplear el título propuesto por Anthony Cárdenas Libro del saber de astrologia con criterios mucho mejor fundamentados y más coherentes con la actitud de Alfonso X y el carácter de las obras que lo integran (Cárdenas, «A New Title», «Hacia una edición»).

27 Cf. Luis Miguel Vicente «El Libro conplido

28 Véase en este sentido el artículo de Lola Ferré. «Los judíos transmisores y receptores de la sabiduría medieval» Revista Española de Filosofia Medieval. Universidad de Zaragoza, vol. 7, 2000, pp. 81-95

29 Cf. Wedel 49. 
nasterio de Ripoll, en el siglo X, donde por primera vez entran en contacto la ciencia occidental con la árabe. Millás Vallicrosa analiza los tratados atribuidos a Gerberto, en los que se encuentran ideas sobre la construcción de astrolabios, cuadrantes y relojes, además de teorías árabes sobre la esfera; en su estudio edita, a modo de apéndice, un tratado sobre el uso del astrolabio, traducción de Llobet de Barcelona y otros textos de materia semejante y pone de manifiesto la relación de estos manuscritos de Ripoll con otros europeos. ${ }^{30}$ Hacia fines del siglo XI y principios del XII las traducciones se incrementan y se pasa de una etapa anónima a la aparición de unos traductores con personalidad muy definida como el judío converso Mosé Sefardí de Huesca, que se bautizó con el nombre de Pedro Alfonso y cuya traducción Disciplina Clericalis supone la entrada del cuento oriental en Occidente. ${ }^{31}$ Pedro Alfonso tradujo además libros de astronomía que revolucionaron el saber en la Europa cristiana. A principios del XI Mosé Sefardí viajó por toda Europa y sobre todo por Inglaterra, por donde difundió por vez primera la ciencia astrológica y matemática que los árabes habían desarrollado; en Inglaterra hizo discipulos; entre ellos Abelardo de Bath, Walcher de Malvern y otros. S. Gil sitúa a Abelardo de Bath en España hacia 1126, y da como causa de su venida la posible amistad con Mosé Sefardí que le había conocido durante su estancia en Inglaterra; habría venido a España buscando las tablas de al-Huwarizmi, que eran difíciles de encontrar en Europa porque estaban basadas en el meridiano de Córdoba (30). Abrahan bar Hiyya ha-Bargeloní o el Barcelonés fue el primer intérprete de la nueva ciencia para los reinos del norte de España y sur de Francia, que no conocían bien el árabe. Colaboró con Platón de Tívoli con quien tradujo diversas obras científicas árabes y alguna hebraica. Las traducciones científicas de el Barcelonés influyeron a toda Europa durante la Edad Media y el Renacimiento. En el noroeste de España y el sur de Francia se fundaron escuelas para trasmitir la cultura árabe en Europa. En esos centros, R. Abraham Ibn Ezra - Avenarius o Abraham Judaeus en su nombre latino- ejerció una gran influencia en la primera mitad deI siglo XII. Nacido a finales del siglo XI en Tudela, Ibn Ezra se dedicó sobre todo a las ciencias, aunque su cultura, como en el caso de otros astrólogos, era enciclopédica. Actuó como el trasmisor de la nueva ciencia tanto para los judíos como para los cristianos de la Península. Escribió en hebreo pero también en bajo latín medieval para la población cristiana, pues esta lengua le servía, además, para viajar por Europa. Entre sus obras astronómicas destacan la Fundamenta tabularun astronomicarum, y un tratado sobre el astrolabio; sus tratados astrológicos se difundieron por Europa profusamente. ${ }^{32} \mathrm{~A}$ él se debe, según el ilustre hebraísta Millás Vallicrosa, el abandono incipiente de la tradición neoplatónica, que había dominado entre los judíos y árabes en los siglos X y XI, para seguir el aristotelismo, cuyo apogeo estará representado por Maimónides, en quien Santo Tomás se inspira no poco. Ambos tuvieron la misma pretensión de hacer compatibles ciencia y fe, cada uno desde su credo respectivo. A través de los estudios de Millás Vallicrosa conocemos también cómo la literatura hebrea incorporó pronto los saberes astrológicos. ${ }^{33}$ Este uso literario de la astrología debió ser un

30 Sobre la importancia de los monasterios hispánicos en esta época precoz para la difusión de la ciencia árabe en Occidente, Vernet suministra datos y bibliografía específica en su Histoira 73 y ss.

31 Un esclarecedor artículo sobre la contribución de Pedro Alfonso y Raymond de Marsella para la aceptación de la nueva astrología árabe, es el de John Tolan. «Reading God’s will in the stars. Petrus Alfonsi and Raimond de Marseille defend the new Arabic Astrology» Revista de Fiosofia Medival. Universidad de Zaragoza, vol. 7, 2000, pp.13-31.

32 Cf. Millás Vallicrosa 134.

33 Millás Vallicrosa nos da ejemplos de esta adaptación literaria de conceptos astronómicos en la poesía hebrea:

Introdujéronme mis pensamientos, oh Dios, a considerarte, mostráronme, en los ojos del alma, tus maravillas, me persuadieron a manifestar tus prodigios, puesto que contemplo tus cielos, obra de tus manos. 
ejemplo para los autores cristianos. La propia inseguridad judía después de la invasión almohade y la conquista de Jerusalén por las cruzadas, debió ser no poco acicate, como Millás Vallicrosa propone, para que surgiera entre ellos una expectación mesiánica que se traduciría en cálculos para establecer el fin de la dominación musulmana y la venida de un Mesías (97).

José S. Gil ha estudiado el componente judío de la Escuela de Traductores de Toledo tanto en la época de las traducciones latinas como en las castellanas; en su trabajo ofrece una idea de la importancia sin par del elemento judío para la trasmisión de la nueva ciencia al resto de Europa. Los judíos contribuyeron de un modo decisivo para que la astrología en Occidente renaciera del vacío a que había quedado relegada durante la baja latinidad. Ya desde el siglo XII se la considera como la más importante de las artes liberales y adquirirá todavía más gloria durante el siglo XIII, transformándose en la ciencia dominante y líder de las demás; junto a ella, de un modo difícilmente separable, se encumbraría igualmente la astrología: «L'astronomie devient de plus en plus la science dominante [...] du fait de son caractère de science pilote [...] aussi comme constituant la partie théorique d'une discipline dont l'autre face est tout naturellement l'astrologie» (Beaujouan «Une lente» 855). La astrología recibirá la atención y el patrocinio de las más importantes figuras del panorama cultural del Occidente cristiano, entre ellas, en lugar preeminente, la atención de Santo Tomás por el lado de la Iglesia, y la de Alfonso X el Sabio por la parte del poder político.

\section{SANTO TOMÁS}

No puede entenderse la actitud de Santo Tomás hacia la astrología considerándolo solamente dentro de la tradición patrística. A diferencia de los otros Santos Padres, santo Tomás escribió en una época —el siglo XIII- en la que las traducciones de la Escuela de Traductores de Toledo sobre todo, habían puesto a disposición del mundo occidental cristiano obras no sólo astrológicas sino también clásicas de máxima importancia. Con el vacío de conocimiento creado después de la caída del Imperio Romano, los escritores cristianos de la Edad Media sólo contaban, como nos dice Menéndez y Pelayo, con unas pocas obras clásicas: el Timeo de Platón, algunos tratados lógicos de Aristóteles, las compilaciones de Casiodoro, Beda, San Isidoro y Alcuino, algunos libros de Séneca y Apuleyo, y la l sagoge de Porfirio y poco más. ${ }^{34}$ Con las traducciones del árabe durante los siglos XII y XIII, Santo Tomás tuvo acceso a las obras que más afectaron su postura ante la astrología, sobre todo los libros de Aristóteles, Platón, Ptolomeo y, por el lado judío, a los tratados Avicebrón y Maimónides que anticipan muchas de sus soluciones al problema.

Santo Tomás en general sigue la concepción de la estructura del Universo expresada por Aristóteles aunque, en cuanto al problema de la astrología y el libre albedrío sigue a mi ver más de cerca a Plotino o a Avicebrón. El santo dominico se enfrenta a la concepción ptolemaica del

La rueda de los cielos camina sobre su órbita, cumpliendo su revolución alrededor de su eje, y narrando, sin labios, tu gloria, mientras la tierra permanece en medio, suspendida con las cuerdas de tu amor. Allí asciende el sol y resplandece, y comunica su luz a la luna, mientras el firmamento es como tienda abierta, y sus estrellas son como un arriate florido para anunciar la profundidad de tus pensamientos.

(Mose ibn Ezra, nacido en Granada hacia 1055), (Literatura 88).

34 Cf. Menéndez Pelayo Heterodoxos 2175. 
Universo y de otros astrólogos árabes y judíos. Como la mayoría de los autores de la antiguiedad, Santo Tomás acepta la división entre el mundo sublunar, compuesto de los cuatro elementos, y el supralunar, integrado por los cuerpos celestes que están formados por un quinto elemento o quintaesencia, como había propuesto Aristóteles. También acepta del filósofo peripatético el concebir el Universo como una serie de esferas planetarias concéntricas en torno a la tierra y envueltas, a su vez, por una esfera más alta e inmóvil que contiene las estrellas fijas. $\mathrm{El}$ mundo de los cuerpos celestes no está sometido a corrupción como el terrestre en virtud de esa quinta esencia de que está formado, y origina, a través de sus movimientos regulares y jerarquizados, los cambios del mundo sublunar. En cuanto al problema de las influencias planetarias en los hombres, Santo Tomás se plantea una solución, sobre todo en las «Quaestio 115 y 116» de la Summa Theologiae, que va a culminar los esfuerzos de la Iglesia por hacer compatibles ciencia y fe. Santo Tomás enmarca el problema de las influencias planetarias dentro de la cuestión más general de actione corporalis creaturae, o sea, dentro del problema de si la materia tiene poder de causar actividad.

En contra de lo que en general sostienen los platónicos - sobre todo Avicebrón- Santo Tomás sostiene que lo material actúa en tanto que está en acto, y actúa sobre otras cosas materiales en tanto que éstas están en potencia: «Dicendum est ergo quod corpus agit secundum quod est actu, in aliud corpus secundum quod est in potentia» (93). El problema de los cuerpos celestes lo discute Santo Tomás a partir de ciertas premisas sobre las que construye sus silogismos; en pocas palabras, asume que las cosas que más están sometidas a cambio derivan sus movimientos de las cosas que están menos sometidas a cambio: «Et ideo quanto aliqua magis sunt immobilia tanto sunt magis causa eorum quae sunt magis mobilia» (100). Así pues, como para Aristóteles, los cuerpos celestes son los que menos cambian ya que sólo se mueven los planetas en sus ciclos regulares, frente al movimiento multidireccional de las cosas terrestres. Los planetas son la causa de los cambios que se operan en la región sublunar:

Corpora autem caelestia sunt inter alia corpora magis immovilia; non enim moventur nisi motu locali. Et ideo motus horum inferiorum corporum, qui sunt varii et multiformes, reducuntur in motus corporis caelestis sicut in causam (102).

Los cuerpos celestes no son la causa primera de las trasformaciones de las cosas, como sostienen los que consideran dioses a los planetas - «quod corpora caelestia non sunt prima causa generatinis et corruptionis eorum que hic fiunt; sicut dicebant illi qui ponebant corpora caelestia esse deos» (102) - sino que los planetas son para Santo Tomás, como para Aristóteles, una especie de instrumentalización del cambio; unos instrumentos que, cambiando ellos mismos en menor grado, constituyen una especie de fuente de las transformaciones que se hacen más numerosos conforme se desciende hacia el mundo sublunar:

Unde secundum Philosophum, necesse est ponere aliquod principium activum mobile, quod per suam praesentia et absentiam causet varietatem circa generationem et corruptionem inferiorom corporum. Et hujusmodi sunt corpora caelestia. Et ideo quidquid in itis inferioribus generat, et movet ad speciem est sicut instrumentum caelestis corporis, secumdum quod dicitur in Physic, quod homo generat hominem, et sol (1153).

A diferencia de las cosas terrestres que forman multitud de especies, los cuerpos celestes son iguales en especie, pero contienen, por su virtud universal, lo que se genera en los cuerpos inferiores:

Ad tertium dicendum quod actiones corpora caelestia inferioribus corporibus non sunt similia similitudine speciei, sed inquantum sua universali virtute continent in se quidquid in inferioribus generatur (1153). 
Los cuerpos inferiores reciben la actividad de los cuerpos celestes de acuerdo a sus propias características materiales, de modo que, para Santo Tomás, como para San Agustín a quien cita, un cuerpo del mundo inferior puede no estar dispuesto para recibir una influencia planetaria tal como la «masculinidad,» por ejemplo, y disponerse más, en cambio a la «feminidad» por sus propias características. Esta posible inadecuación de los cuerpos terrestres a recibir una influencia planetaria había sido utilizada, como Santo Tomás nos informa, por San Agustín como argumento para negar la ciencia de la adivinación a través de las estrellas:

As quartum dicendum quod actiones corporum caelestium diversimodi recipintur in inferioribus corporibus secundum diversam materiae dispositionem. Contigit autem quandoque quod materia conceptus humani non est dispositia totalitier ad masculinum sexum; unde partim formatur in masculum, partim in feminam. Unde et hoc introducitur ab Agustino ad repellendum scilicet divinationem quae fit per astra; quia effectus astrorum variantur etiam in rebus corporeis, secundum diversam materiae dispositionem (1153).

Sin embargo, fuera de esa variable capacidad que la materia tiene de recibir las influencias planetarias, para Santo Tomás el hecho es que el mundo de la materia es influido directa y esencialmente por los cuerpos celestes como establece en la cuestión 115 4: «Dicendum quod corpora caelestia in corpora quidem imprimunt directe e per se» (104). Como consecuencia de esa influencia en el cuerpo, los planetas pueden afectar indirectamente incluso el intelecto y la voluntad humanas, en la medida en que el intelecto o la voluntad se dejen influir por el cuerpo:

Sciendum est tamen quod indirecte et per accidens impressiones corporum caelestium ad intellectum et coluntatem pertinere possunt, inquantum scilicet tam intellectus quam voIuntas aliquo modo ab inferioribus viribus accipiunt, quae organis corporeis alligantur (106).

De esas dos cualidades, el intelecto es más susceptible que la voluntad a las influencias planetarias ya que el primero es más impresionable por los sentidos mientras que la voluntad puede librarse de la influencia de los deseos bajos $\longrightarrow$ o mundo sensorial- y no seguir el dictado de las pasiones, que es lo que faciliataría la influencia de los planetas en la voluntad humana, como lo establece en la cuestión 115 5:

Nam intellectus ex necesitate accipit ab inferioribus viribus apprehensivis; unde turbata vi imaginativa vel cogitativa vel memorativa, ex necessitate turbatur actio intellectus. Sed voluntas non ex necessitate sequitur inclinationem appetitus inferiores, licent enim passiones quae sunt in irascibile et concupiscililli, habeant quandam vim ad inclinandam voluntatem; tamen in potestate voluntatis remanet sequi passiones vel eas refutare (106).

Esa será la síntesis del pensamiento cristiano sobre la astrología y las influencias planetarias: el hombre es libre de actuar en dirección del bien o del mal porque tiene una voluntad que le permite sustraerse a las influencias del mundo material - determinadas por los planetas. En virtud de esa voluntad, el hombre no puede negar su responsabilidad para salvarse o condenarse. Los planetas en ningún caso son los responsables por sí mismos, como materia, de las influencias en el intelecto del hombre; sino que, instrumentos ellos mismos, como se ha visto, están movidos por «sustancias espirituales» que afectan al intelecto en forma de iluminación, pero que no pueden impedir el libre ejercicio de la voluntad, como lo pondera Santo Tomás en la cuestión 115 :

Ad primum ergo dicendum quod spirituales substatiae quae caelestia corpora movent in corporalia quidem agunt mediantibus caelestibus corporibus, sed in intellectum humanum agunt immediate, illuminando. Voluntatem autem immutarenon possunt, ut supram habitum est (106). 
No obstante, como la mayoría de los hombres siguen el dictado de sus pasiones, la astrología puede en la mayoría de los casos también, predecir el futuro de esos hombres, aunque no el de los sabios que, exactamente igual que decía Plotino, por el ejercicio de su voluntad o libre albedrío, están capacitados para controlar sus pasiones y escapar así a toda predicción basada en los cuerpos celestes:

Ad tertium dicendum quod plures hominum sequuntur passiones, quae sunt motus sensitivi appetitus, ad quas cooperari possunt corpora caelestia; pauci autem sunt sapiens, qui hujusmodi passionibus resistant. Et ideo astrologi ut in pluribus vera possunt praedicere, et maxime in communi. Non autem in speciali, quia nihil prohibet aliquem hominem per liberum arbitrium passionibus resitere. Unde et ipsi astrologi [Ptolomeo] dicunt quod sapiens homo dominatur astris, inquantum scilicet dominatur suis passionibus (106).

Esta es la cuestión decisiva que afecta a la actitud de la Iglesia respecto a la astrología; actitud que se reflejará en el pensamiento y la literatura medievales constantemente.

Por otro lado, no todo lo que pasa en la tierra tiene que ver con el influjo de los astros, sino que ocurren una serie de cosas por accidente o por fortuna que no pueden atribuirse a la influencia de las estrellas sino sólo a la Providencia, como defiende Santo Tomás en la cuestión 116 1:

Impossibile est ergo quod aliqua virtus activa caelestis corporis sit causa eorum quae hic aguntur per accidens, sive a casu sive a fortuna. Et ideo dicendum est quod ea quae hic per accidens aguntur, sive in rebus naturalibus sive humanis, reducuntur in aliquam causam praedominatem: quae est providentia divina (118).

Relacionado con el influjo de los planetas en los hombres, trata Santo Tomás el problema de la cooperación de los demonios con los cuerpos celestes. Para él, la naturaleza de los demonios y la de los planetas es distinta: los demonios son sustancias espirituales y por lo tanto -al carecer de materia — no pueden estar bajo la acción de los planetas: «[...] dicimus enim daemones esse substantias intellectuales corporibus non unitas. Unde patet quod non subduntur actione caelestium corporum, nec per se nec per accidens, nec directe nec indirecte» (108). Lo que ocurre es que los demonios para perjudicar a los hombres y difamar la creación de Dios - los planetas son parte de esa creación- actúan considerando el momento más propicio para vencer la voluntad de los hombres, lo cual lógicamente ejecutan cuando las influencias planetarias hacen al ser humano más proclive a seguir sus pasiones, usando, por ejemplo, el influjo de la Luna sobre la parte húmeda del cerebro que inclina a los hombres — sobre todo a los lunáticos-a sus pasiones:

Ad primum ergo dicendum quod hoc quod daemones secumdum certa augmenta lunae homines vexant contingit propter duo. Primo quidem ad hoc quod infament creaturam Dei, scilicet lunam, ut Hieronymus et Chrysostomus dicunt. Secundo, quia cum non possint operari nisi mediantibus naturalibus virtutibus [...], in suis operibus considerant corporum aptitudines ad effectus intentos. manifestum est autem quod cerebrum humisissimum est omnium partium corporis, ut Aristoteles dicit, et ideo maxime subjicitur operationi lunaem, quae ex sui proprietate habet movere humorem (110).

Siendo así que la mayoría de los hombres siguen sus pasiones, que la astrología en esa medida puede predecir sus destinos y que, además, los mismos demonios, conociendo cómo influyen los planetas en las inclinaciones pasionales de los hombres, pueden coadyuvar con los influjos naturales para conducir al hombre al pecado, hará falta la ayuda divina para que esa mayoría de hombres «débiles» pueda salvarse. Para eso están los seres divinos o santificados del mundo cristiano con cuya ayuda, como tanto se repetirá en la literatura castellana medieval, el hombre puede salvarse. Consciente de los peligros a los que el hombre común está ex- 
puesto, Santo Tomás dedica toda una serie de «Quaestiones» de su Summa Theologiae a definir y enfatizar la ayuda que Dios, a través de los seres que le sirven, como los ángeles, proporciona al hombre para que pueda salvarse cuando no sean suficientes el libre albedrío y el conocimiento universal que inclina al ser humano al bien; así lo determina en la cuestión 113 1:

Ad primun ergo, dicendum quod per liberum arbitrium potest homo aliqualiter malum vitare, sed non sufficienter; quia infirmatur circa affectum boni propter multiplices animae passiones. Similiter etiam universalis cognitio naturalis legis, quae homini naturaliter adest, aliqualiter diriget hominem ad bonum, sed non sufficienter, quia in applicando universalia principia juris ad particularia opera, contigit hominem multipliceterdeficere. Unde dicitur Sa., Cogitationes mortlium timidae, et incertae providentiae nostrae. Et ideo necessaria fuit homini custodia angeli (50).

En suma, Santo Tomás expresó una áctitud conciliadora entre el legado tradicional de la patrística de carácter radicalmente opuesto a la astrología y la presión de la nueva filosofía que emanaba de las obras astrológicas y filosóficas fruto de las traducciones de obras científicas árabes, muchas de las cuales se hicieron en el escritorio alfonsí, ${ }^{35}$ y que reclamaban una actitud más dialogante por parte de la Iglesia para no quedarse totalmente en frente o al margen de las teorías científicas de la época, cuya inspiración, de manos de la astrología, seguía siendo holística y lo seguiría siendo hasta la llegada del racionalismo cartesiano.

\section{BIBLIOGRAFÍA}

Al-Biruni. The Book of Instruction in the Elements of Astrology. Ed. y trad. de R. Ramsay Wright. London: Luzac, 1934.

Amador de los Ríos, José. Estudios históricos, políticos y literarios sobre los judios de España. Buenos Aires: Ediciones Argentinas Solar, 1942.

Baër, Yitzhak. Die Juden im Christlichen Spanien. 2 tomos. Berlin: Akademie fur die Wissenschaft des Judensthums, 1929-1936.

Beaujouan, Guy. «Une lente préparation au 'decollage' des sciences (quadrivium et médicine) dans la France de Philippe-Auguste.» La France de Philippe-Auguste: le temps des mutations. Actes du Colloque International organisé par le CNRS. Tomo 602. (29 septembre-4 octobre) Paris: 1980. 852853.

— «L'enseignement du quadrivium.» Settimane di studio del Centro Italiano di Studi sull' alto medioevo. (Spoleto) 19 (1972): 123-195.

— «L'Astronomie dans la péninsule Ibérique à la fin du Moyen Age.» Agrupamento de estudos de cartografia antiga. Coimbra, 1969.

Cantera Burgos, F. El judío salmantino Abraham Zacut. Notas para la historia de la Astronomía en la España medieval. Madrid: C. Bermejo, Impresor, s. f.

Cardenas, Anthony. «A New Title for the Alfonsine Omnibus on Astronomical Instruments.» La Corónica 8 (1980): 172-177.

Carmody, Francis J. Arabic Astronomical and Astrological Sciences in Latin Translation. A critical Bibliography. Berkeley: University of California Press, 1956.

Cruz Hernández, Miguel. Historia del pensamiento en el mundo islámico. 2 tomos. Madrid: Alianza Editorial, 1981.

Duhem, Pierre. Le système du monde, histoire des doctrines cosmologiques, de Platón a Copernic. 10 tomos. Paris: Librairie Scientifique A. Hermann et Fils, 1913-1959.

Durand, Gilbert. «L'Astrologie, Langage de l'Unus Mundus.» L'Astrologie. Paris: Editions Albin Michel, 1985. 200-213.

35 Cf. Luis Miguel Vicente. «La Astrología para Alfonso X el Sabio» Actas del Congreso Proyección Histórica de España en sus tres culturas: Castilla y León, América y el Mediterráneo. Junta de Catilla y León, 1993: pp.379-386, Vol. I 
Gil, José S. La Escuela de Traductores de Toledo y sus colaboradores judíos. Toledo: Instituto de Investigaciones y Estudios Toledanos, 1985.

Goldstein, B. R. «The survival of Arabic Astronomy in Hebrew.» Journal for the History of Arabic Science 3 (1979): 31-39.

Iancu-Agou, Danièle. «Astronomie et astronomes juifs dans le Midi de la France au Moyen Age.» Le Soleil, la Lune et les Etoiles au Moyen Age. Provence: Université de Provence, 1983. 195-215.

Ibn 'Ezra, R. Abraham. El libro de los fundamentos de las Tablas alfonsíes. Ed. crít., intr. y notas José María Millás Vallicrosa. Madrid-Barcelona: Consejo Superior de Investigaciones Científicas, 1947.

Kibre, P. «The Quadrivium in the Thirteenth Century Universities, with Special Reference to Paris.» Arts libéraux et Philosophie au moyen âge. Actes du IVe Congrès International de Philosophie Médiévale. Montréal-Paris: 1969.

Lainstner, M. L. W. «The Western Church and Astrology During the Erarly Middle Ages.» The Harvard Theological Review. 34 (Oct.1941): 251-276.

Lemay, Richard. «The True Place of Astrology in Medieval Science and Philosophy: Towards a Definition.» Astrology, Science and Society. Historical Essays. Ed. Patrick Curry. New Hampshire: The Boydell Press, 1987. 57-76.

Millás Vallicrosa, José M. Assaig d'història de les idees fisiques i matemàtiques a la Catalunya medieval. Tomo 1. Barcelona: Institución Patxot, 1931.

— «El literalismo de los traductores de la Corte de Alfonso el Sabio.» Al-Andalus. 1 (1933): 155-188.

- Estudios sobre la historia de la ciencia española. Barcelona: Consejo Superior de Investigaciones Científicas, 1949.

- Las traducciones orientales en los manuscritos de la Biblioteca Catedral de Toledo. Madrid: Consejo Superior de Investigaciones Científcas, 1942.

- Literatura hebraicoespañola. Barcelona: Labor, 1967.

- Nuevas aportaciones para el estudio de la transmisión de la ciencia en Europa a través de España. Barcelona: Consejo Superior de Investigaciones Científicas, 1943.

- Nuevos Estudios sobre historia de la ciencia española, Barcelona: Consejo Superior de Investigaciones Científicas, 1960.

North, J. D. «Medieval Concepts of Celestial Influence. A survey.» Astrology, Science and Society. Historical Essays. Ed. Patrick Curry. New Hampshire: The Boydell Press, 1987. 5-16.

Pérès, Henri. Esplendor de al-Andalus. La poesía andaluza en árabe clásico en el siglo XI. Sus aspectos generales, sus principales temas y su valor documental. Madrid: Ediciones Hiperión, 1983.

Phares, Simon de. Recuil des plus célèbres astrologues et quelques hommes doctes. Ed. Ernest Wickersheimer. Paris: 1929.

Préaud, Maxime. Les astrologues à la fin de Moyen Age. Paris: J. C. Lattès, 1984.

Rico, Francisco. El pequeño mundo del hombre. Madrid: Castalia, 1970.

Salvat, Michel. «Barthemi l'Anglais, Traites du Soleil et de la Lune, traduits par Jean Corbechon (1372) (édition et commentaire).» Le Soleil, la Lune et les Etoiles au Moyen Age. Provence: Université de Provence, 1983. 339-357.

Sams6, Julio. «The Early Development of Astrology in al-Andalus.» Journal for the History of Arabic Science 3 (1979): 228-243.

- «Alfonso X y los orígenes de la astrología hispánica.» Estudios sobre la historia de la ciencia árabe. Editados por Juan Vernet: Barcelona: 1980: 83-114.

— «Dos notas sobre astrología medieval.» Al-Andalus 36 (1971): 215-222.

Tallgren, O. J. «Sur l'astronomie espagnole d'Alphonse X et son modèle arabe.» Studia Orientalia 1 (1925): 342-346.

Thorndike, Lynn and Kibbe, P. A Catalogue of Incipits of Mediaeval Scientific Writtigs in Latin. Cambridge, Mass.: Mediaeval Academy of America, 1937.

Thorndike, Lynn. A History of Magic and Experimental Science During the First Thirteen Centuries of Our Era. 8 tomos. New York: The Ferris Printing Company, 1923-58.

Tolan, John. «Reading God's will in the stars. Petrus Alfonsi and Raimond de Marseille defend the new Arabic Astrology» Revista de Fiosofia Medival. Universidad de Zaragoza, vol. 7, 2000: 13-31.

Tomás de Aquino, Santo. Summa Theologiae Texto latino e inglés. (10: «Cosmogony.» Ed. William.A. Wallace); (11: «Man.» Ed. Timothy Suttor); (15: «The World Order.» Ed. M. J. Charlesworth). LondonNew York: Blackfriars, Eyre \& Spottiswoode y McGraw-Hill Book Co., 1970.

Torroja Martínez, José María. El sistema del mundo desde la Antigüedad hasta Alfonso X el Sabio. Madrid: Instituto de España, 1980. 
Vadet, Jean-Claude. «Quelques observations sur la pratique de l'astrologie chez les Arabes.» L'Astrologie. Paris: Editons Albin Michel, 1985. 49-78.

Van de Vyver, E. «Les plus anciennes traductions latines médiévales (Xe-XIe siècles) de traités d'astronomie et d'astrologie,» Osiris 1 (1936): 658-691.

Vernet Ginés, Juan. Historia de la ciencia española. Madrid: Instituto de España, Cátedra <<Alfonso X el Sabio $\gg, 1975$.

- La ciencia en Al-Andalus. Sevilla: Biblioteca de Cultura Andaluza, 1986.

- La cultura hispanoárabe en Oriente y Occidente. Barcelona: Ariel, 1978.

—, ed. Nuevos estudios sobre astronomía española en el siglo de Alfonso $X>$ Barcelona: Universidad Autónoma, Facultad de Filosofía y Letras, 1983.

-, ed. Textos y estudios sobre astronomía en el siglo XIII. Barcelona: Universidad Autónoma, Facultad de Filosofía y Letras, 1981.

Vicente García, Luis Miguel. «La Astrología para Alfonso X el Sabio» Actas del Congreso

Proyección Histórica de España en sus tres culturas: Castilla y León, América y el Mediterráneo. Junta de Castilla y León, 1993: 379-386, Vol. I

— «San Agustín, San Gregorio y San Isidoro ante el problema de las estrellas:

fundamentos para el rechazo frontal de la Astrología» Revista Española de Filosofia Medieval. Universidad de Zaragoza, 2001: 187-205. Vol.8

— «Plotino y el problema de las estrellas: una solución para los neoplatónicos» Revista Española de $\mathrm{Fi}$ losofia Medieval. Universidad de Zaragoza, 2000: 191-198. Vol. 7

Wedel, Theodore Otto. The Mediaeval Attitude Toward Astrology, Particularly in England. New Haven: Yale University Press, 1920.

Luis Miguel Vicente García Universidad Autónoma de Madrid

Facultad de Filosofía y Letras, Departamento de Filología Española Ciudad Universitaria de Cantoblanco 28049 Madrid email: luismiguel.vicente@uam.es 